\title{
PAPER
}

\section{Integrating top-down/bottom-up sustainability strategies: an ethical challenge}

\author{
John Cairns, Jr.* \\ Department of Biology, 1020 Derring Hall, Virginia Polytechnic Institute and State University, \\ Blacksburg, Virginia 24061, USA
}

\begin{abstract}
Sustainable use of the planet will require multiple sustainability strategies, which will range from the entire system, the entire Earth, to the local or regional. Strategies starting at the highest system level are referred to as 'top-down,' and strategies designed for components, local or regional, are referred to as 'bottom-up.' Doubtless, several intermediate levels will eventually be required, although the number is far from clear at this time. It is abundantly clear that both top-down and bottom-up strategies must be integrated effectively or neither will work well. Furthermore, there will be significant uncertainties at both levels of organization, which will be reduced as evidence accumulates. However, sustainability is too complex and dynamic to reduce scientific uncertainty to a level desired by most decision makers. A greater emphasis on sustain-ethics and value judgments will improve communications between those working at different organizational levels since humankind's wish to leave a habitable planet for its descendants and those of other life forms is clearly a value judgment.
\end{abstract}

KEY WORDS: Top-down strategy $\cdot$ Bottom-up strategy $\cdot$ Sustainability strategies $\cdot$ Eco-ethics $\cdot$ Value judgments

Resale or republication not permitted without written consent of the publisher

Knowledge of what is does not open the door directly to what should be.

Albert Einstein

I know of no more encouraging fact than the unquestionable ability of man to elevate his life by conscious endeavor. Henry David Thoreau

\section{INTRODUCTION}

The Options Spring 2002 issue is devoted entirely to Achieving Sustainable Development: The 21st Century Imperative. Jernelöv's (2002) editorial notes that human security issues relating to the supply of water, food, and energy, and the protection of Earth's life support systems have been high on the International Institute for Applied Systems Analysis' priority list since its creation approximately three decades ago. The same Options issue notes that human dimensions must be placed at the core of sustainable development to meet the needs of present generations without sacrificing the livelihoods of future generations. Not featured is eco-ethics - the ethics of humankind's relationship with the planet's biospheric life support system, despite the fact that the natural capital and services it provides is the sine qua non of sustainability.

Sustainable use of the planet is the most complex problem in human history. Its goal is to change humankind's behavior and practices so that the human species can inhabit its planet indefinitely. To accomplish this goal, it is essential that a mutualistic relationship develop between human society and natural systems. This relationship, in turn, will require a combination of econ-ethics and eco-ethics (e.g. Kinne 2002). Both economics and ecology are derived from the Greek word oikos, which means household. The 
term household was originally used (and still is) in a much more restricted way; however, humankind is now beginning to perceive Earth as the ultimate household. Econ-ethics requires an ethical economic system that will benefit humankind. The economy must be structured in a way that will not damage the planet's ecological life support system. Econ-ethics and eco-ethics used concomitantly to enhance sustainable use of the planet would be sustain-ethics (this term initiated in this paper). Neither natural systems nor future generations can demonstrate appreciation or gratitude for sustain-ethics while present generations still live, but ethical behavior gives a peace of mind that is its own reward. However, sustain-ethics, in addition to compassion for natural systems and future generations, also includes compassion for disadvantaged members of the human species who could express appreciation to humans now alive for improving the human condition.

There must be a global strategy for sustainability ('top-down strategy') but also a strategy that considers the unique issues and ecosystems of each bioregion ('bottom-up strategy'). Holistically practicing topdown and bottom-up sustainability strategies, including several intermediate 'connecting' levels, is a formidable, daunting task. The most promising way to connect these interdependent activities is an ethical 'cement' - sustain-ethics. It is unclear whether the topdown strategies should work directly with the bottomup strategies or whether there should be one or more intermediate steps. Ideally, the shorter the communication chain the more rapid and effective communication will be, but there are many obstacles to this simple two-strategy model.

The lofty goals of sustainability are fairly easily stated and seem to strike a responsive chord in anyone wishing future generations of humankind to have a habitable planet. How this will be implemented in various ecoregions with different problems is not particularly clear; even less clear is the way in which different ecoregions will interact with each other and how the humans who occupy them will be persuaded to follow a global sustainability strategy.

It is abundantly clear that both top-down and bottom-up strategies are being developed, although the rate of development of the latter varies dramatically from one country to another and from one bioregion to another. In addition, global acceptance of whatever top-down strategy eventually emerges will doubtless be markedly influenced by local conditions.

Both global and regional strategies must be integrated effectively or neither will work well. Humankind has only one finite planet, and damage to one part of it almost guarantees damage to other parts that are distant geographically, spatially, and even temporally.
It remains to be seen whether humans can grasp a problem of such complexity for an infinite period of time. After all, sustainable use means being able to continue these practices indefinitely. However, if it is not possible to address effectively a problem of such complexity, humankind will suffer enormously. Therefore, the attempt must be made despite many inherent difficulties.

\section{OBSTACLES TO TOP-DOWN/BOTTOM-UP SUSTAINABILITY STRATEGIES}

The obstacles to developing a sustainability initiative are essentially the same as those hampering development of a global sense of community. Even taken individually, they both have inherently formidable obstacles. In the aggregate, one wonders how they will ever be transcended. Illustrative examples of obstacles to bottom-up strategy development include: (1) language barriers, (2) ethnic and religious conflicts, (3) disparities in per capita wealth, (4) disparities in educational opportunities, particularly in developing scientific and environmental literacy, (5) differences in the balance between individualism and a sense of community, (6) differences in age distribution within the local population (e.g. predominantly young or predominantly elderly), (7) level of biophilia (humankind's innate affinity for the natural world), (8) degree of compassion for individuals of the human species distant in time (future generations) or space (in far geographic localities), (9) level of equity and fairness in resource allocation among members of the human species and those of other life forms, and (10) a lack of willingness to do more than the law requires in achieving sustainable use of the planet. Illustrations of obstacle 10 include focus on individual 'rights' (Cairns 2002a,b) rather than individual responsibilities, lack of accountability for one's own actions, greedy desire to acquire material wealth at others' expense, disregard for the appearance of one's environment (e.g. littering in public parks and freeways), corporate efforts to obtain today's profits without regard for the long-term effects of today's practices, elected officials' actions geared toward satisfying constituents' immediate demands (to keep the vote) instead of doing what is best for the survival of the local ecosystem, and the waste of resources that is a part of affluent societies' consumerism.

The obstacles to development of a top-down strategy are equally formidable. Illustrative examples include: (1) the enormous difficulty in visualizing solutions to goals over large temporal and spatial spans, (2) integrating huge temporal and spatial spans in a mutualistic fashion, (3) ensuring that no component of the topdown strategy negates or compromises an important 
component of the bottom-up strategy, (4) developing continuous feed-back loops between top-down and bottom-up strategies, (5) developing a harmonious working relationship between top-down and bottomup strategists, (6) ensuring that minutia do not distract from the holistic scope of the top-down strategy, (7) detecting changes in either natural systems or human society that require mid-course corrections, (8) coping with 'rogue' nations and uncooperative nations in an ethical way while maintaining sustainable practices, (9) acquiring and maintaining the financial base necessary to operate the Global Sustainability Organization (GSO), (10) determining when the precautionary principle should be applied, and (11) determining how to orchestrate the GSO in a democratic fashion while promptly eliminating unsustainable practices.

Clearly, the same obstacles exist for both top-down and bottom-up - they will, however, not be resolved in an identical fashion. These illustrative examples do indicate how important ethics will be for both strategies. As humankind moves from small group, tribal units toward a global community, shared ethical values become ever more important to the survival of the human species.

\section{UNCERTAINTY AND ETHICS}

There are four major classes of scientific uncertainty, particularly in resolving environmental issues such as sustainability: (1) framing uncertainty, (2) modeling uncertainty, (3) statistical uncertainty, and (4) decision-theoretic uncertainty (Shrader-Frechette 1996).

Durham (1992) remarks that scientists provisionally accept a hypothesis that has survived rigorous attempts to falsify it, even one with obvious deficiencies, if there is no better (i.e. more probable) hypothesis available. Physics has often been regarded as one of the 'hardest' sciences with a superb record of validating hypotheses. Yet, Carnap (1966) states that since hypotheses have an infinite number of observational consequences that can never be conclusively validated, scientists sometimes opt, in an uncertain situation, for provisional acceptance of the best available non-falsified hypothesis. Thus, uncertainty is the norm, even within disciplines noted for their precision.

Uncertainty is likely to be orders of magnitude greater in the quest for sustainable use of the planet, which requires input from all disciplines. Further, experimentation is difficult, not only because there is only one planet but also because of a natural reluctance to experiment with human subjects. Cairns \& Smith (1996) analyze some of the ways in which these uncertainties associated with both top-down and bottom-up strategies can be reduced and make recom- mendations on how the validation process might belatedly be integrated into the ecotoxicological field. These approaches should be useful, properly modified, when integrating top-down and bottom-up approaches in general. As Cairns \& Smith (1996) note, it is difficult (and in some cases impossible) to measure directly how a stressor affects an ecosystem. For example, society cannot wait 20 or more years to determine the specifics of the ecological effects of radioactive wastes. While this uncertainty is being reduced, ethical principles will be a useful component of societal decisions. Ethical principles are extremely important since scientific uncertainty may never be satisfactorily reduced in time periods of interest to human society.

Sustainable use of the planet is an aspiration involving levels of complexity transcending most scientific endeavors. Arguably, one of the most important facets of this complex problem is that most environmental laws and regulations place the burden of proof for demonstrating human health or environmental damage on governmental regulatory agencies or nongovernmental organizations wishing to demonstrate harm from development or technological activities. The universal standard, which is generally used to meet burden of proof requirements, is often the normal standard of scientific proof, such as a $95 \%$ confidence level or an equivalent criterion. The scientific community, in order to minimize Type I errors and, therefore reduce speculation in scientific data interpretation, adopted this standard. But when such a standard is utilized as a basis for developing sustainability strategies, the scientific uncertainty that inevitably pervades such situations means that the burden of proof usually is not met, despite the fact that some information might demonstrate impairment to the quest for sustainability. Finally, as a consequence of the absence of a robust understanding of scientific uncertainty and its implications for sustainability, decisions mean that policymakers/managers will not have adequate scientific information to guide them in terms of whether or to what extent decisions should reflect a precautionary approach. Since humankind has only one finite planet on which to achieve sustainable use, it is abundantly clear that the usual requirements to reduce scientific uncertainty, such as use of controls, multiple testing under variable conditions, and the like, cannot be met. Ethics are especially important in such circumstances.

Humankind is now moving from the age of reductionist science to an age of synthesis or integrative science. This transition does not mean that reductionist science is no longer appropriate, but rather that as levels of complexity in any system increase, new properties emerge that were not apparent at lower levels. Consequently, one means of reducing uncertainty in this age of synthesis is how congruent a particular 
hypothesis or body of evidence is with other related bodies of evidence within the particular system being studied. Both top-down and bottom-up sustainability strategies will require synthesis and also a means of coping with scientific uncertainty. Again, ethics should be a major factor in the decision making process.

\section{POLICYMAKERS/MANAGERS AND SOCIAL AND NATURAL SCIENTISTS}

It is well to remember that all sustainability strategies involve both macro- and micro-coevolution of human societies and natural systems. If successful sustainability strategies are developed, they will also require coevolution in understanding between and among policymakers/managers and natural and social scientists. These coevolutionary interactions will greatly influence sustainability issues, as well as issues of how humans behave toward the environment, those in other cultures and financial circumstances. Most importantly, it will require abandoning the many unsustainable practices found in almost every culture on the planet and substituting sustainable practices despite the attractiveness of the unsustainable ones to which humans have become accustomed. These are groups unaccustomed to working together on a longterm, meaningful basis on such a complex issue as sustainability strategies. At worst, some groups have no regard for or even trust in some of the other groups, and, at best, there is often a poor understanding of the ways in which other groups function. Ethics is an obvious 'bridge' between groups so that misunderstandings can be reduced or eliminated.

One would expect the primary initiative in developing integrative programs covering the broad spectrum of groups just described to come from the world's universities and colleges, particularly those in which the responsibility for generating new knowledge and communicating it to students is a major responsibility. Regrettably, this does not occur at either the rate or scale necessary for achieving sustainability for a variety of reasons. Among these are increased teaching loads and budget cuts at state- and federallysupported institutions, which do not permit the extensive time necessary for faculty in one discipline to develop a deep understanding and productive relationships with those in other disciplines. Arguably most importantly, students do not perceive the need for developing such a broad perspective because they cannot, at present, see the relationship to the job market and their future professional growth. Consequently, it is highly unlikely that there will be adequate, experienced personnel skilled in integration and synthesis at the temporal and spatial scales required for sustainability initiatives as well as the diversity of components needed for successful implementation. Such personnel cannot be produced overnight, nor is it likely that many persons with a disciplinary bias can be persuaded to take a holistic view of sustainability initiatives. As a consequence, it will be of greatest importance to utilize the relatively few available personnel as effectively as possible in the short term and to prepare a much larger group that is sufficiently holistic to implement sustainability initiatives. The most important aspect of educational institutions' budget cuts is fewer personnel and less scientific information for a considerable period of time. Uncertainty will not be significantly reduced and may even increase. Thus, ethics is now of major importance.

\section{HOW MANY LEVELS OF SUSTAINABILITY STRATEGIES?}

Going from global to local or regional directly follows Dubos' famous injunction 'think globally, act locally.' The problem is that insightful global thinking will require an information mass well beyond the capability of most (and possibly all) individuals to assimilate and understand. Even if an individual, or even a small number of individuals, did have such a capability, there would be a problem of trust because they would undoubtedly not represent all religions, all cultures, all language groups, and so on. Furthermore, since effective sustainability strategy implementation will involve numerous professional and non-professional groups and a mixture of science and value judgments, a diversity of viewpoints would strengthen the policy decisions, if the diversity did not impede reaching consensus. In the first two decades of the 21st century, it is extremely unlikely that there would be adequate numbers of competent personnel to function as integrators of concepts and information and synthesizers of both concepts and value judgments. If there is a global public will to increase the number of competent professionals, undoubtedly this could be done over a period of several decades or more. The initial problem would be the lack of suitable faculty and other professionals to educate and inform the large numbers of additional personnel needed. Furthermore, much onthe-job training would be required, and a large number of qualified personnel would be required to spend most, if not all, of their time on synthesis and information integration rather than on increasing the literacy of additional personnel.

At the bottom-up level, there are numerous areas where adequate or nearly adequate numbers of competent professionals are available. There are also numerous areas where the idea of sustainability is 
not even being discussed in the most general way. So, there is a major educational problem at the bottom-up level, although in most respects it differs significantly from the top-down approach. A major problem in increasing literacy at the bottom-up level concerns trying to increase all citizens' literacy in the requirements for sustainable use of the planet and deciding what organization(s) should be responsible for quality control, planning, financing, and the like. There is also the crucial question of how to transfer increased sustainability literacy from areas where the literacy is high to areas where it is low or nonexistent. This problem will almost certainly be exacerbated by cultural, religious, and language difficulties, to name just a few. Ethics should help reduce these problems because it may furnish a common ground in which diversity can be appreciated but not divisive. Kung (1998) defines a comprehensive ethic-founded on the bedrock of mutual respect and humane treatment of all beings-that would encompass the ecological, legal, technological, and social patterns that are reshaping civilization. If humans are going to have a global economy, a global media, a global technology, Kung (1998) argues that there must also be global ethics to which all nations and peoples of the most varied backgrounds and beliefs can commit themselves. Earth can and should be held together by ethics. As Common (1995) notes, there is no purely scientific basis on which to decide the alternative positions between economists and ecologists. Differences primarily reflect dissimilar value systems (e.g. Myers \& Simon 1994). This is one reason why sustainability issue decisions are so difficult and contentious. It is abundantly clear that the debate on sustainability issues would be more productive if participants explicitly stated their ethical values that, together with scientific evidence, support the positions they are taking.

At the outset, there seems to be no choice but to begin with only the top-down approach and the bottom-up approach with no intermediate stages. This design will undoubtedly cause difficulties, but these will doubtless be less if there are competent personnel in both categories rather than a large number of unqualified people at intermediate organizational stages or, worse yet, at all organizational stages. This immediately calls to mind the problem of quality control, which has been successfully resolved by the disciplines representing reductionist science but has yet to be resolved for integrative science or synthesis.

As the number of qualified personnel increases and the general literacy about sustainability increases, more levels of organization between top-down and bottom-up not only will be possible but most likely essential.

\section{CONCLUSIONS}

For initial stages, the primary focus of sustainability initiatives should be restricted to top-down and bottom-up strategies with no intermediate levels, for reasons already stated. As the number of trained personnel and the information base, including case histories, expands, so also can the number of intermediate stages between the two extremes. Exacerbating the complex problems already discussed in a preliminary fashion will be the certainty that there is no precise indication of how much time is left to put these topdown/bottom-up strategies in place. Many professionals think environmental problems are already severe and that a number of crucial environmental thresholds and breakpoints have already been crossed. Resilient systems usually permit an overshoot if it is not too severe and not sustained for an exceptional time period. However, many unsustainable practices are increasing exponentially while social adjustments lag far behind. Possibly, it will require a major collapse of one of the planet's life support systems to change the mood from complacency to serious concern. This also would mean that the time to cope with the problem and to increase sustainability literacy will be substantially decreased. Consequently, one hopes that reason guided by evidence will result in some precautionary measures being taken, such as increasing training programs at universities and colleges and, as soon as possible, in general school systems, which will enable all citizens to become literate in this area.

Clearly, a greater emphasis on ethics and value judgments with regard to sustainable use of the planet is long overdue. Science can show what probably is done; technology can show what might be done; but ethics can help humankind decide what should be done.

Acknowledgements. I am deeply indebted to Eva M. Call for transcribing the dictation of the first draft of this manuscript and to Darla Donald for editorial work in preparing it for publication. The Cairns Foundation paid for processing costs.

\section{LITERATURE CITED}

Cairns J Jr (2002a) Reexamining the 'inherent worth and dignity of every person' paradigm in an interdependent web of life context. J Lib Rel 3(1), available at www.meadville.edu/cairns_3_1.html

Cairns J Jr (2002b) Revisiting respect for the interdependent web of life and the worth and dignity of each individual: a major issue in sustainable use of the planet. Common Ground 1.2 (2002):29-34

Cairns J Jr, Smith EP (1996) Uncertainties associated with extrapolating from toxicological responses in laboratory 
systems to the responses in natural systems. In: Lemons J (ed) Scientific uncertainty and environmental problemsolving. Blackwell, Cambridge, MA

Carnap R (1966) Philosophical foundation of physics. Basic Books, New York

Common M (1995) Sustainability and global policy. Cambridge University Press, Cambridge

Durham P (1992) The aim and structure of physical theory. Trans. Wiener P. Princeton University Press, Princeton, NJ Jernelöv A (2002) Editorial. Options (Spring 2002):1

Editorial responsibility: Mary Batson (Managing Editor) Oldendorf/Luhe, Germany
Kinne O (2003) Eco-ethics further developed text: 01.05.2002. EEIU Brochure, Inter-Research, Oldendorf/Luhe

Kung H (1998) A global ethic for global politics and economics. Oxford University Press, Oxford

Myers N, Simon J (1994) Scarcity or abundance: a debate on the environment. Cambridge University Press, Cambridge

Shrader-Frechette K (1996) Methodological rules for four classes of scientific uncertainty. In: Lemons J (ed) Scientific uncertainty and environmental problemsolving. Blackwell, Cambridge, MA

Submitted: December 19, 2002; Accepted: February 3, 2003 Proofs received from author(s): February 6, 2003

Published on the web: February 6, 2003 